\title{
3D trajectories and velocities of rainfall drops in a multifractal turbulent wind field
}

\author{
Auguste Gires ${ }^{1}$, Ioulia Tchiguirinskaia ${ }^{1}$, and Daniel Schertzer ${ }^{1}$ \\ ${ }^{1}$ Hydrologie Meteorologie et Complexite (HM\&Co), Ecole des Ponts Paris-Tech, Champs-sur-Marne, France \\ Correspondence: Auguste Gires (auguste.gires@enpc.fr)
}

\begin{abstract}
Weather radars measure rainfall in altitude whereas hydro-meteorologists are mainly interested in rainfall at ground level. During their fall, drops are advected by the wind which affects the location of the measured field.

The governing equation of a rain drop motion relates the acceleration to the forces of gravity and buoyancy along with the drag force. It depends non-linearly on the instantaneous relative velocity between the drop and the local wind; which yields 5 to complex behaviour. Here, the drag force is expressed in a standard way with the help of a drag coefficient expressed as a function of Reynolds number. Corrections accounting for the oblateness of drops greater than 1-2 mm are suggested and validated through comparison of retrieved "terminal fall velocity" (i.e. without wind) with commonly used relationships in the literature.

An explicit numerical scheme then is implemented to solve this equation for 3+1D turbulent wind field, and hence analyse the temporal evolution of the velocities and trajectories of rain drops during their fall. It appears that multifractal features of the input wind are simply transferred to drop velocity with an additional fractional integration whose level depends on drop size, and a slight time shift. Using actual high resolution 3D sonic anemometer and a scale invariant approach to simulate realistic fluctuations of wind in space, trajectories of drop of various size falling form $1500 \mathrm{~m}$ are studied. For a strong wind event, drops located within a radar gate in altitude during $5 \mathrm{~min}$ are spread on the ground over an area of size few kilometers. Spread for drops of a given diameter are found to cover few radar pixels. Consequences on measurements of hydro-meteorological extremes which are needed to improve resilience of urban areas are discussed.
\end{abstract}

\section{Introduction}

During their fall, drops are advected by wind. Quantitative rainfall estimation with the help of weather radars are affected by this issue since drops can be displaced horizontally between their measurement location in altitude and their ground impact location which is of interest for hydro-meteorologists. This effect is usually called wind drift in the literature and sometimes wind advection. The potential bias and uncertainty introduced in radar measurement is stronger at higher resolution, i.e. typically with pixel size smaller than 1-2 $\mathrm{km}^{2}$ which are needed for urban applications for example. Collier (1999) suggests that 
correction schemes should be implemented for this kind or higher radar resolution. Lauri et al. (2012) reported that far from radar (i.e. typically more than $150 \mathrm{~km}$ ), even with low elevation $\left(0.3^{\circ}\right)$, displacements of few tens of $k m$ are found, which actually distort the measured area.

Most correction schemes rely on the use of 4D wind profiles derived from numerical predictions models (Mittermaier et al., 2004; Lack and Fox, 2007; Lauri et al., 2012; Sandford, 2015) or combination of such with reanalysis (Dai et al., 2013, 2019). The latter also accounts for drop size distribution (DSD). With such, they report an improvement by $\approx 3 \%$ of the correlation between radar and rain gauge measurement and a reduction of discrepancy of $\approx 18 \%$ over eight selected events. Lack and Fox (2007) used directly Doppler radar wind measurement at $2.5 \mathrm{~km}$ scale to adjust for wind drift effect. In general, correction schemes use wind data at rather coarse resolution (typically $\mathrm{km}(\mathrm{s})$ ) and assume a constant wind shear. Nevertheless, some variability at smaller space-time scales is usually acknowledged, especially during convective events, i.e. the ones for which wind drift causes the greatest uncertainty (Lack and Fox, 2007).

Wind effects on rainfall drops is also reported to generate discrepancies between measured vertical velocities and expected terminal fall ones. For example, Montero-Martinez and Garcia-Garcia (2016) studied events with calm, light and moderate wind with various rainfall levels, and found a widening of the fall velocity distribution under windy conditions. For example, they found super-terminal drops only for diameters $<0.7 \mathrm{~mm}$ and more often under wind conditions. Sub-terminal fall velocities for drops of size up to $2 \mathrm{~mm}$ are reported. Bringi et al. (2018) found that under low wind speed and turbulence, no discrepancies are found with expectations while under high wind speed and turbulence, there is a clear widening of the distribution. A linear decrease of mean fall velocity with increasing turbulent intensity is reported. Maximum decreases of 25-30\% are observed. Thurai et al. (2019) also found such decrease for drops greater than $2 \mathrm{~mm}$ in high turbulence intensity conditions. It is associated to an asymmetry also appearing in the drop shape. They also found that drop horizontal velocities in both direction and magnitude show "remarkable agreement" with the wind sensor at $10 \mathrm{~m}$. Stout et al. (1995) explored the effect of non linear drag coefficient on fall velocity through numerical simulations. They showed that even heavy drops exhibited a reduced settling velocity in isotropic turbulence.

Turbulence is found to have contradictory effects on the distribution of fall velocity. Indeed increasing turbulence level in windy and rainfall condition will yield to more collision and breakup, resulting in smaller drops inheriting the speed of larger parent ones, hence observations of super-terminal velocities. On the other end, turbulence is said to yield to a decrease in fall velocities because drops (especially ones $<1 \mathrm{~mm}$ ) are more affected by eddies.

Such findings on the discrepancies between observed and expected fall velocities has effects on the relation between rainfall and kinetic energy, i.e. the erositivity 'power' of rainfall (Pedersen and Hasholt, 1995) and also building performance to outdoor conditions (Tian et al., 2018; Blocken et al., 2011).

The studies previously mentioned basically do not account for small scales wind fluctuations in both space and time. In this paper, we suggest to study the behaviour of individual rainfall drops of various sizes in a high resolution turbulent wind field. The variability of the wind is accounted for through the framework of Universal Multifractals (UM) (see Schertzer and Tchiguirinskaia, 2020, for a recent review). Such physically based framework is designed to analyse and simulate geophysical fields exhibiting extreme variability over wide range of space-time scales as wind. Drop oblateness is also accounted for. 
The paper is organized as follow. In section 2, a deterministic equation for the fall of oblate drops in a 3D field is derived and validated through the comparison of obtained terminal fall velocity with commonly used formulas. In section 3 , the framework of Universal Multifractals is briefly reminded. Then, the drops are subjected to simulated multifractal fields as wind input and multifractal behaviour of horizontal drop velocity is assessed. Finally, in section 4, 3D wind is reconstructed from high resolution 3D sonic anemometer data and strong scaling assumptions. This field is used to study the trajectories of drops between falling from $1500 \mathrm{~m}$ down to the ground.

\section{A deterministic equation for oblate drops in a wind field}

\subsection{Formulation of the equation}

Let us denote $(\mathrm{x}, \mathrm{y}, \mathrm{z})$ the horizontal, lateral and vertical coordinates in a standard Cartesian framework with unit vectors $\left(\underline{e}_{x}, \underline{e}_{y}, \underline{e}_{z}\right)$. We aim at writing the motion equation of a particle of water (a drop) of velocity $\underline{v}_{p}$, density $\rho_{p}$ and falling in the atmosphere under the influence of the gravity $\underline{g}=-g \underline{e}_{z}$ (where $g=9.81 \mathrm{m.s} \mathrm{s}^{-2}$ ) and the wind $\underline{v}_{\text {wind }}$. The density of the atmosphere is denoted $\rho_{\text {air }}$. The water particle is characterized by its equivolomic diameter $D_{e q}$ which corresponds to the diameter of the sphere having the same total volume. Hence we have $V o l=\frac{\pi}{6} D_{e q}^{3}$. Finally the relative velocity between the wind and the falling particle is $\underline{v}_{r e l}=\underline{v}_{\text {wind }}-\underline{v}_{p}$.

The drop is subjected to three forces :

- The gravity equal to $\rho_{p}$ Volg

- The buoyancy equal to $-\rho_{\text {air }} V$ olg

- The drag, which is commonly written as $\frac{1}{2} \frac{\pi D^{2}}{4} c_{D} \rho_{a i r} v_{r e l} \underline{v}_{r e l}$. Re is the common Reynolds number $\operatorname{Re}=\frac{\rho_{a i r} v_{r e l} D}{\mu_{a i r}}$ where $\mu_{a i r}$ is the absolute viscosity of air. $c_{D}$ is the drag coefficient and depends in general of $R e$ and $D_{e q}$. The next section is devoted to its determination.

As a consequence, the equation of motion of the falling particle is :

$80 \frac{d \underline{v}_{p}}{d t}=\frac{3}{4 D} c_{D} \rho_{a i r} v_{r e l} \underline{v}_{r e l}+\underline{g} \frac{\rho_{p}-\rho_{a i r}}{\rho_{p}}$

\subsection{Determination of the drag coefficient}

Before discussing how the drag coefficient is determined, it should be reminded that rainfall drops which are considered in this paper are not spherical. Indeed drops greater than typically $1.5 \mathrm{~mm}$ become oblate in their fall. This oblateness increases with size. A very commonly used model consists in an ellipsoid with an axis ratio varying depending on the size. Thurai et al. (2007) showed that such model is too simplistic since drops are not symmetric in the direction perpendicular to their fall. Following 
an in-depth analysis of the drop shape assessed with the help of a 2D-video disdrometer (Kruger and Krajewski, 2002) in the measurement campaign of an artificial rainfall experiment; they suggested the following formula for the shape :

$x=c_{1} \sqrt{1-\left(\frac{z}{c_{2}}\right)^{2}}\left[\cos ^{-1}\left(\frac{z}{c_{2} c_{3}}\right)\right]\left[c_{4}\left(\frac{z}{c_{2}}\right)^{2}+1\right]=f(z)$

with :

$$
\begin{aligned}
& c_{1}=\quad \frac{1}{\pi}\left(0.02914 D_{e q}^{2}+0.9263 D_{e q}+0.07791\right) \\
& c_{2}=\quad-0.01938 D_{e q}^{2}+0.4698 D_{e q}+0.09538 \\
& 90 \quad c_{3}=-0.06123 D_{e q}^{3}+1.3880 D_{e q}^{2}-10.41 D_{e q}^{2}+28.34 \\
& c_{4}=-0.01352 D_{e q}^{3}+0.2014 D_{e q}^{2}-0.8964 D_{e q}^{2}+1.226 \text { if } D_{e q}>4 \mathrm{~mm} \\
& c_{4}=\quad 0 \text { if } 1.5 \mathrm{~mm} \leq D_{\text {eq }} \leq 4 \mathrm{~mm}
\end{aligned}
$$

This shape corresponding to a solid of revolution around $z$ axis is used in this paper. It is displayed in Fig. 1.a for drops with equivolumic diameter ranging from $1.5 \mathrm{~mm}$ to $5.5 \mathrm{~mm}$. It should be mentioned that computing the volume as an integral of the shape ( $V o l=\int_{z_{\min }}^{z_{\max }} \pi f(z)^{2} d z$ ) yields to minor differences with the expected volume of $\frac{\pi D_{e q}^{3}}{6}$. They are highlighted in Fig. 1.b. As a consequence once an equivolumic diameter is set, the corresponding one that would lead to the expected volume from Eq. 2 and 3 is computed from a correspondence table. The relationship, which is obviously close to the bisector is displayed in Fig. 1.c. A consequence is that oblateness of drops will be considered only from equivolumic diameter greater than $1.527 \mathrm{~mm}$

For non spherical shapes, it is quite tricky to compute the corresponding drag coefficient as a function of the Reynolds number. The literature about this issue is quite abundant and the interested reader is referred to chapter 4 of the $\mathrm{PhD}$ dissertation of Baheri (2015) or Hölzer and Sommerfeld (2008) for details. In the approach they implemented, three parameters are used to characterize the non spherical shapes of the falling particle with the help of three dimensionless parameters: The sphericity, the crosswise sphericity and the lengthwise sphericity. The two last depend on the orientation of the particle with regards to the flow. Here it is assumed that drops are oriented perpendicularly to the flow, i.e. the 'z' axis of Eq. 2 is parallel to $\underline{v}_{r e l}$. In the general case, these parameters may be complex to assess but with the shape derived from Eq. 2 (Thurai et al., 2007), theoretical formula can be obtained. The three parameters are :

- The sphericity $\psi$ which is equal to ratio between the surface area of the equivolumic sphere to the actual surface area of the particle. It is equal to one for sphere and decreases for less and less spherical particles. $\psi=\frac{p i D_{e q}^{2}}{\text { Surface Area }}$. In the framework of this paper, i.e. we have $S A=\int_{z_{\min }}^{z_{\max }} 2 \pi f(z) \sqrt{1+f^{\prime}(z)^{2}} d z$

- The crosswise sphericity $\psi_{\perp}$ which is equal to ratio between the projected area of the volume equivalent sphere and the projected area of the particle normal to the falling direction (here $\underline{e}_{z}$ ). $\psi_{\perp}=\frac{D_{e q}^{2}}{D_{m a x}^{2}}$. It is equal to one for sphere and decreases for larger drops since they become oblate. 

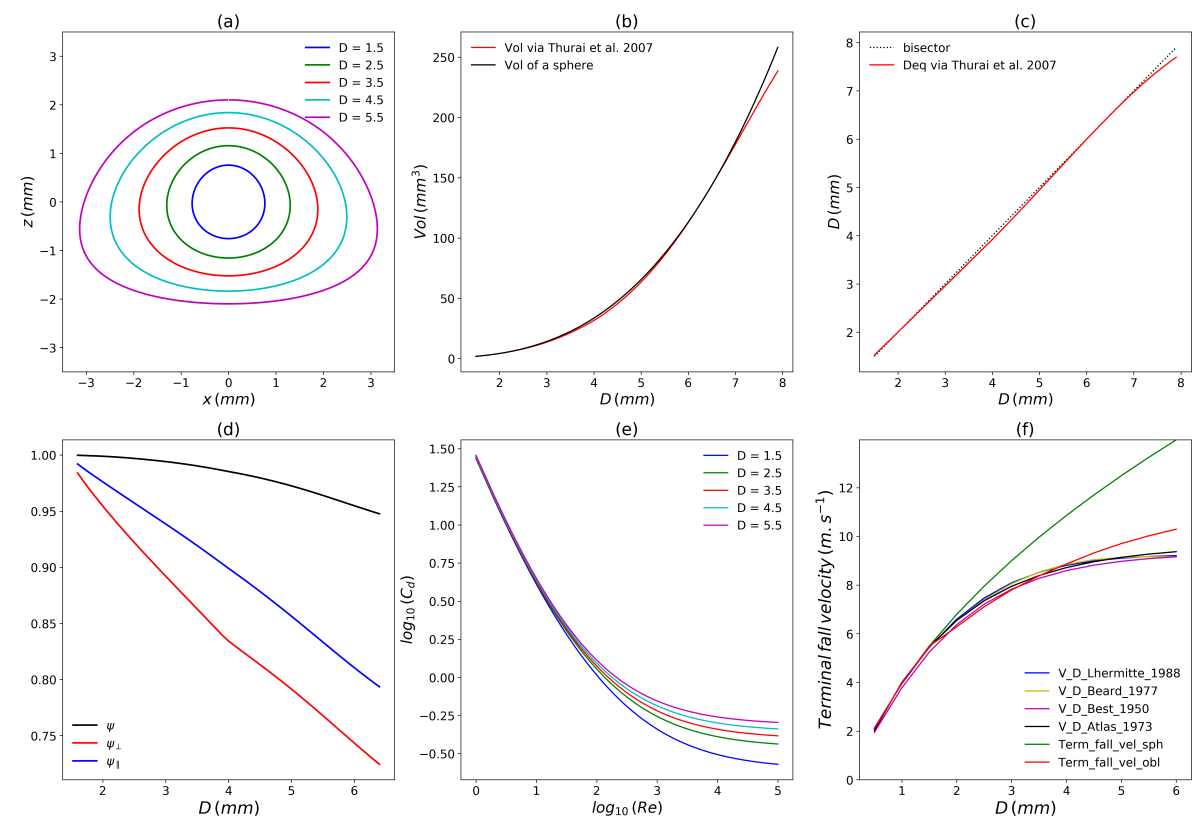

Figure 1. (a) Drop shape model used in this paper; (b) Drop volume vs. equivolumic diameter; (c) Diameter relation to retrieved wanted volume; (d) Parameters characterizing non spherical shape of drops vs. equivolumic diameter; (e) Drag coefficient $C_{d}$ vs. $R e$ number; (e) Terminal fall velocity vs. equivolumic diameter

- The lengthwise sphericity $\psi_{\|}$which is defined as to the cross-sectional area of the volume equivalent sphere divided by the difference between half the surface area and the mean projected longitudinal cross-sectional area of particle $\left(M P A_{\|}\right)$. $\psi_{\|}=\frac{\pi\left(\frac{D_{e q}}{2}\right)^{2}}{\frac{S A}{2}-M P A_{\|}}$. In the specific drop model of this paper, we have $M P A_{\|}=\int_{z_{\min }}^{z_{\max }} 2 f(z) d z$.

The evolution of these parameters as a function of $D_{e q}$ for the considered drops is in Fig. 1.d. The increasing oblateness of drops with increasing size is translated through the fact that the parameters are getting further away from 1 . In order to define the drag coefficient, the corrections suggested by Hölzer and Sommerfeld (2008) to account for non sphericity of particles are then implemented on the formula of White (1974) previously used by Stout et al. (1995) who worked only on spherical drops. This yields :

$C_{D}=\frac{8}{R e \sqrt{\psi_{\|}}}+\frac{16}{R e \sqrt{\psi_{\perp}}}+\frac{6}{(1+\sqrt{R e}) \psi^{3 / 4}}+\frac{0.25 \times 10^{0.4(-\log \psi)^{0.2}}}{\psi_{\perp}}$

The evolution of $C_{D}$ as a function of $R e$ for various drop parameters is displayed in Fig. 1.e and follows standard patterns.

\subsection{Validation of the formula}

In order to validate the developed equation, the retrieved terminal fall velocity is assessed for each equivolumic diameter. It corresponds to the velocity of the permanent regime with no wind, i.e. the drag plus the buoyancy exactly compensate the 
gravity. Computations are carried out with $\rho_{\text {air }}=1.205 \mathrm{~kg} \cdot \mathrm{m}^{-3}, \mu_{\text {air }}=1.8110^{-5} \mathrm{~kg} \cdot \mathrm{m}^{-1} . \mathrm{m}-2, \rho_{\text {water }}=998.2 \mathrm{~kg} \cdot \mathrm{m}^{-3}$ $g=9.81 \mathrm{~m} . \mathrm{s}^{-2}$ as in Stout et al. (1995).

The relation between obtained terminal fall velocity vs. equivolumic diameter is displayed in red in Fig. 1.f. The developed equations enables to retrieve commonly used relation (Beard, 1977; Lhermitte, 1988; Best, 1950; Atlas et al., 1973) for drops of diameter up to $4 \mathrm{~mm}$. The deviations found when considering spherical drops (in green) are visible for diameter greater than $2 \mathrm{~mm}$ which highlights the need to account for drop oblateness.

\subsection{Numerical scheme for solving the equation}

Eq. 1 is solved numerically through the implementation of a simple Eulerian numerical scheme. In such framework: (i) a discretisation of time with time step $\Delta t$ is introduced yielding to discrete time steps $t_{n}=n \times \Delta t$ where $n$ is an integer; (ii) we aim at finding an approximation of $\underline{v}_{p}$ at time step $n$ denoted $\underline{v}_{p, n}$; (iii) the first derivative in Eq. 1 is approximated as $\frac{d \underline{v}_{p}}{d t}\left(t_{n}\right) \approx \frac{\underline{v}_{p, n+1}-\underline{v}_{p, n}}{\Delta t}$. This yields to the following equation for the numerical scheme :

$\underline{v}_{p, n+1}=\underline{v}_{p, n}+\Delta t\left[\frac{3}{4 D} c_{D, n} \rho_{a i r} v_{r e l, n} \underline{v}_{r e l, n}+\underline{g} \frac{\rho_{p}-\rho_{a i r}}{\rho_{p}}\right]$

where $\underline{v}_{r e l, n}$ and $c_{D, n}$ are computed at time step $t_{n}$ using the formulas discussed in section 2.1 and 2.2. Assuming some initial conditions (always no horizontal velocity and a vertical one equal to the terminal fall for the corresponding diameter), it is then possible to reconstruct the time series of velocity for the drops. From it, the temporal evolution of the position (i.e. the trajectory) is derived. It is needed to properly assess the wind accounting for the current position of the drop. A time step of $\Delta=0.01 s$ is used in this paper, and it was checked that it ensured a stability of the numerical scheme.

\section{Behaviour of horizontal drop velocity with multifractal input}

\subsection{Brief reminder on Universal Multifractal framework}

It is outside the scope of the paper to introduce in details the framework of Universal Multifractals (UM). Hence, only the most important elements are reminded here and interested readers are referred to the references mentioned or to a recent review by Schertzer and Tchiguirinskaia Schertzer and Tchiguirinskaia (2020) for more details.

Let us consider a field $\epsilon_{\lambda}$ at a resolution $\lambda$ defined as the ratio between the outer scale $(L)$ and observation scale $(l) ; \lambda=L / l$. For multifractal fields, the moment of order $q$ of the field is power law related to the resolution :

$\left\langle\epsilon_{\lambda}^{q}\right\rangle \approx \lambda^{K(q)}$

where $K(q)$ is the scaling moment function. It fully characterizes the variability across scales of the field. In the specific framework of UM (Schertzer and Lovejoy, 1987, 1997), towards which multiplicative cascades processes converge, only two parameters with physical interpretation are needed to characterize $K(q)$ for conservative fields : 
- $C_{1}$, the mean intermittency co-dimension, which measures the clustering of the (average) intensity at smaller and smaller scales. $C_{1}=0$ for an homogeneous field;

- $\alpha$, the multifractality index $(0 \leq \alpha \leq 2)$, which measures the clustering variability with regards to the intensity level.

For UM, we have :

$K(q)=\frac{C_{1}}{\alpha-1}\left(q^{\alpha}-q\right)$

A non-conservative field $\left(\psi_{\lambda}\right)$, i.e. whose mean is not preserved across scale can be written as $\psi_{\lambda} \approx \epsilon_{\lambda} \lambda^{-H}$, where $H$ is the non-conservativeness parameter. $H=0$ for conservative fields. Positive values correspond to a fractional integration to go from $\epsilon_{\lambda}$ to $\psi_{\lambda}$ and to stronger correlations within the field $\psi_{\lambda}$. Negative values correspond to a fractional differentiation. $H$ is typically between 0 and 1 for geophysical fields.

The first step of a multifractal analysis usually consists in a spectral analysis. For multifractal fields the power spectra $(E)$ should scale with wave number $k$ :

$E(k)=k^{-\beta}$

with the spectral slope $\beta$

$\beta=1+2 H-K_{c}(2)$

where $K_{c}$ is the scaling moment function (Eq. 7) of the conservative part of the field. To analyse the latter, a Trace Moment (TM) is implemented. It notably enables to assess the quality of the scaling behaviour. It basically consists in plotting Eq. 6 in log-log. Straight lines should be retrieved and the slope gives $K(q)$. Finally, UM parameters are estimated with the help of the Double Trace Moment (DTM) technique which is tailored for UM fields and enables robust estimation of UM parameters (Lavallée et al., 1993).

\subsection{Methodology}

In this section, the scaling behaviour of horizontal drop velocity is assessed using numerical simulations. Working with such input whose features are fully known is helpful to understand how drops react to wind.

More precisely, a horizontal input $v_{x, w i n d}$ for Eq. 1 is simulated with the help of blunt multifractal discrete cascades (Gires et al., 2020). Such process yields only positive values which is not realistic for wind. Hence a standard 'complex trick' was used to generate a field with both positive and negative values (Schertzer and Lovejoy, 1995). To implement it, two fields $X_{1}$ and $X_{2}$ are generated with the wanted features, and a third one is obtained with the help of the following equation ( $R e$ is the real part) :

$X=\operatorname{Re}\left[\exp \left(\log X_{1}+i \log X_{2}\right)\right]$

180 Such field divided by two was used as input. 1024 time step long series are generated with UM parameter $\alpha=1.7$ and $C_{1}=0.2$, which corresponds to typical value for turbulent wind fields (Fitton et al., 2011). The time step is assumed to be of $0.01 s$ which 

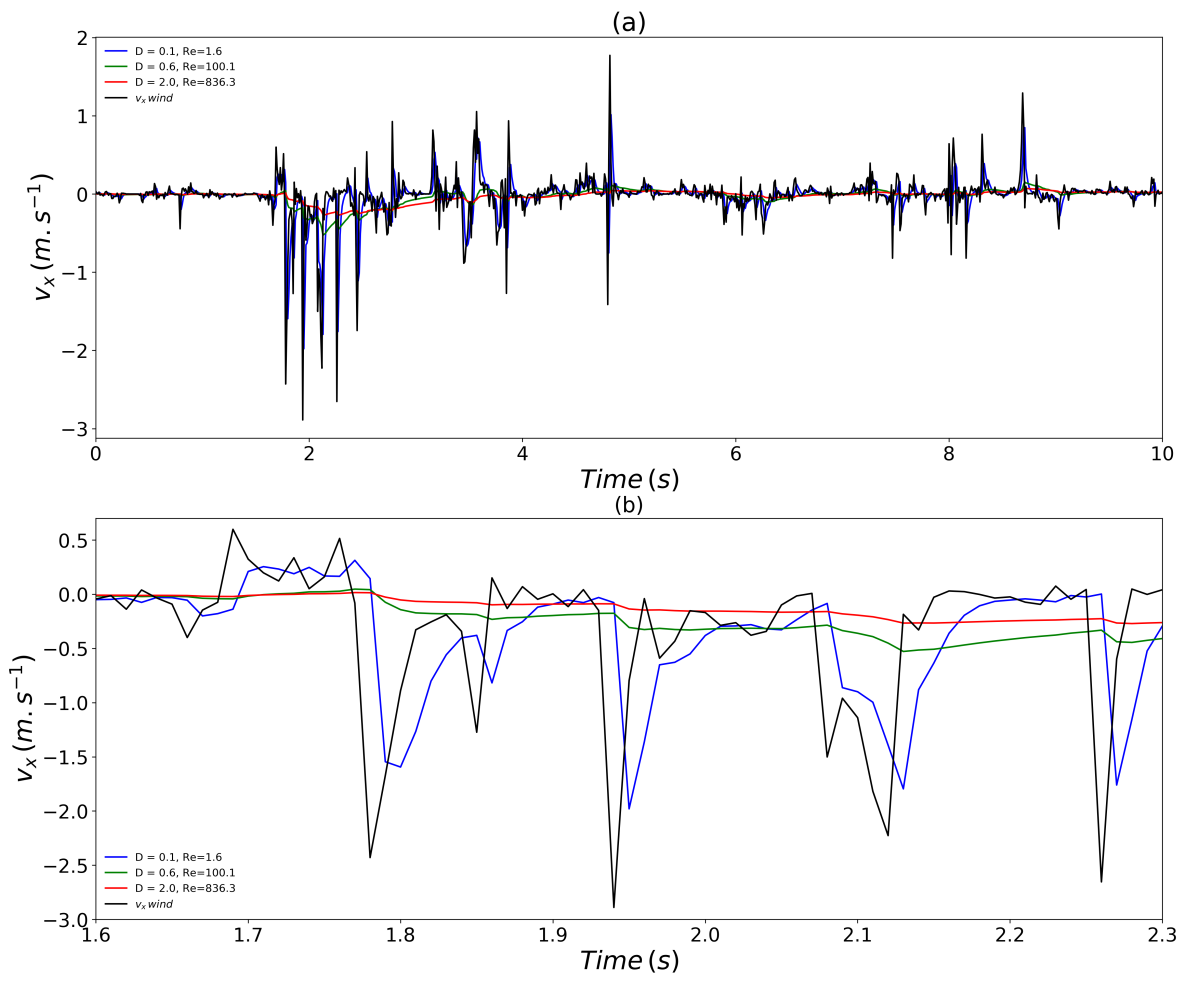

Figure 2. Top : temporal evolution of the drop horizontal velocity during $10 s$ for various drop diameters with the same multifractal wind input (in black). Bottom : a zoom of the above curve on a shorter period

means that drops are basically studied over $10 \mathrm{~s}$. For the initial conditions, drops are assumed to have no horizontal velocity and a vertical component equal to its corresponding terminal fall velocity. Since scaling is a statistical behaviour, an ensemble of 100 independent samples was generated and the corresponding ensemble of horizontal drop velocity was simulated using Eq. 5 for drops of various sized $\left(D_{e q} \subset[0.1,0.2,0.4,0.6,0.8,1,1.5,2,3,4]\right)$

\subsection{Results and discussion}

Fig. 2 displays the temporal evolution of drops' horizontal velocity over $10 s$ for a sample of wind input (in black). Three drop diameters are displayed $(0.1,0.6$ and $2 \mathrm{~mm})$. It can be seen, notably on the zoomed part of the figure (lower panel) that the smaller drop ( $D_{e q}=0.1 \mathrm{~mm}$, in blue) follows well wind fluctuations with only a limited dampening of the fluctuations. A small delay $(\approx 0.01 \mathrm{~s})$ corresponding to a reaction time is noted. As it can be expected larger drops $\left(D_{e q}=0.6 \mathrm{~mm}\right.$, in green; and $D_{e q}=2 \mathrm{~mm}$, in red) tend to dampen even more wind fluctuations.

In order to quantify more precisely this qualitative behaviour, a multifractal analysis on the retrieved ensembles was performed. Fig. 3 displays the outcome of spectral and TM analysis for drops of equivolumic diameter equal to 0.1 and $2 \mathrm{~mm}$. The spectral analysis reflect a good scaling behaviour over the whole range of scales. Spectral slopes ( $\beta$ in Eq. 8) of 0.86 and 2.25 

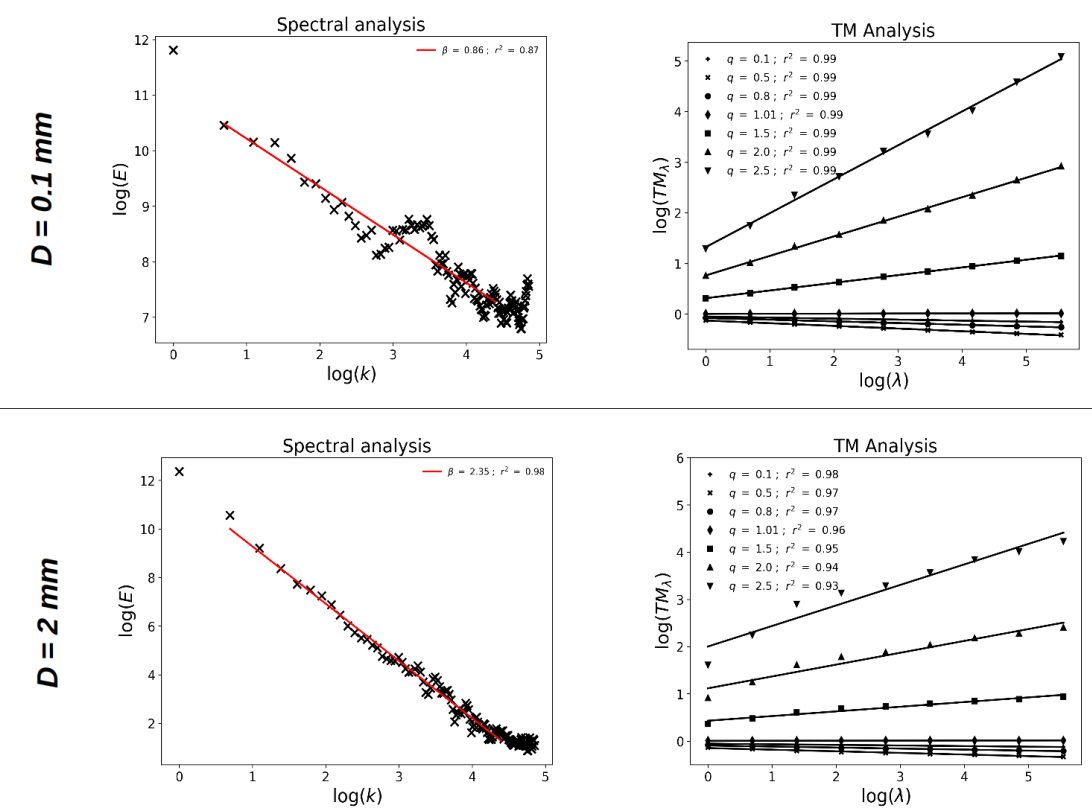

Figure 3. Scaling behaviour of the simulated drop velocity for $D_{e q}$ equal to $0.1 \mathrm{~mm}$ (top row) and $2 \mathrm{~mm}$ (bottom row). Spectral analysis (i.e. Eq. 8 in $\log -\log$ ) on the direct simulations is displayed in the left column. TM analysis (i.e. eq. 6 in log-log) on the velocity after implementing a fractional integration is displayed in the right column

are retrieved respectively. For the $2 \mathrm{~mm}$ drop, the value corresponds to non-conservative fields. In order to ensure that a conservative field is studied in TM analysis, which is needed (Lavallée et al., 1993), a fractional differentiation with an exponent $(\beta-1) / 2$ is implemented on the field before implementing this TM analysis. TM analysis is displayed in the right column of Fig. 3. For the $1 \mathrm{~mm}$ drop, an excellent scaling behaviour is retrieved with coefficient of determination $r^{2}$ for $q=1.5$ greater than 0.99. DTM analysis yields estimates of UM parameters $\alpha, C_{1}$ and $H$ equal to $1.68,0.21$ and 0.12 respectively, which is close to the features of the input series. For the $2 \mathrm{~mm}$ drop, the scaling is slightly degraded but remains good $\left(r^{2}=0.95\right.$ for $q=1.5) . \alpha=1.69, C_{1}=0.14$ and $H=0.79$ is found.

Fig. 4 displays a summary of the UM analysis carried out on the generated series for the various drops. The scaling behaviour is excellent for small drops and remains good for all drop sizes with $r^{2}$ for $q=1.5$ always greater than 0.95 (Fig. 4.e). The need for a fractional differentiation before implementing TM analysis is visible with the very poor scaling found when analysing directly the field. The non-conservativeness parameter rapidly increases from 0.1 to 0.8 with drop size increasing from $0.1 \mathrm{~mm}$ to $\approx 1-1.5 \mathrm{~mm}$. For larger drops it remains rather stable. This increase of $H$ is basically a quantification of the increased dampening of wind fluctuations observed for larger drops discussed with Fig. 2. With regards to the UM parameters $\alpha$ and $C_{1}$, the former remains stable and close to the input value of 1.7 for all drop size. The latter exhibits a small decrease with larger drops. It should be reminded that this approach is somehow artificial since all drops are perceiving the same wind, which would not be the case in reality because they do not fall at the same vertical speed. In summary, this investigation shows that horizontal 

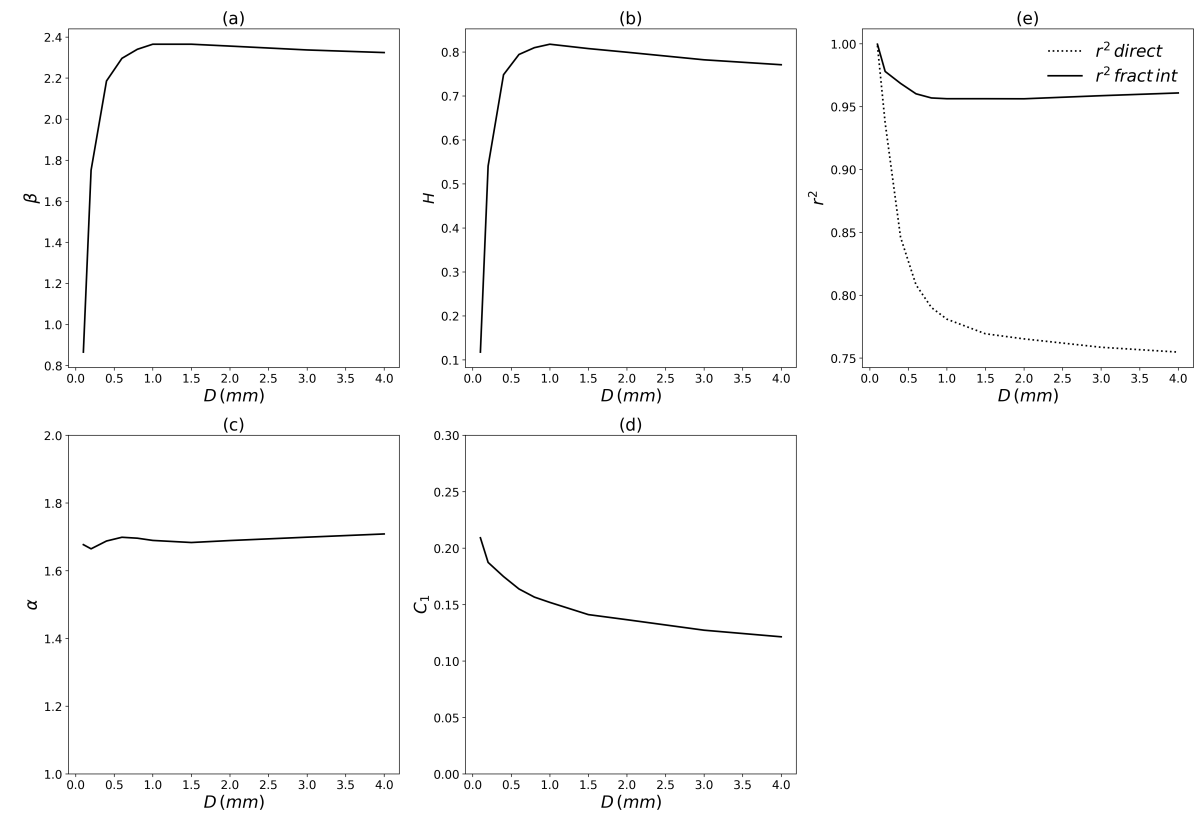

Figure 4. Summary of the multifractal analysis performed on the ensembles of simulated drop horizontal velocities using a wind input with $\alpha=1.7, C_{1}=0.2$. The various multifractal parameters are displayed vs. $D$ (the equivolumic drop diameter)

drop velocity basically reproduces the multifractal properties of the wind input with an increased level of non-conservativeness $H$. $H$ strongly increases for drops smaller than $1 \mathrm{~mm}$ and then stabilizes.

\section{Ground impact location of drops falling in a turbulent wind field}

\subsection{Methodology}

215 The purpose of this section is to investigate where drops falling from a height of $1500 \mathrm{~m}$ reach the ground. Given the time step of $0.01 s$ used in the equation and the fact that drops are moving in space during their fall, it means that having high resolution space-time 3D wind data over an area of typical size few kilometers is needed to fully address the issue. Such data is unfortunately not available. Hence we suggest here to reconstruct a somehow realistic wind from a punctual measurement relying on previous findings on turbulence.

More precisely, we use $100 \mathrm{~Hz}$ 3D sonic anemometer data collected at the Pays d'Othe wind farm in the framework of the ANR RW-Turb project (Gires et al. (2021), Gires et al. 2021 - submitted to HESS). The wind series corresponding to a rather low wind event and a strong one are displayed in Fig. 5 over approx. $900 s$. The low wind event was collected on 20/01/2021 while the strong one occurred on 06/01/2021.

In order to generate a realistic wind at a given distance from the anemometer, standard scaling relations between wind fluctuations and distances are used (Lazarev et al. (1994)). In such framework horizontal wind shifts behave as $\Delta u(\Delta x) \approx$ 


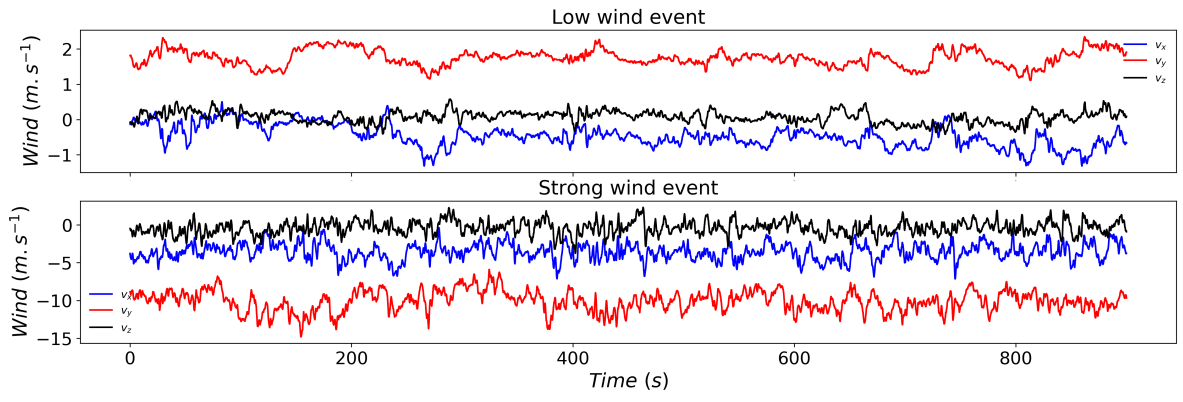

Figure 5. Temporal evolution of the $100 \mathrm{~Hz}$ wind data from 3D sonic anemometer for low (top) and strong (bottom) wind event used in this paper

$\epsilon(\Delta x)^{a_{h}} \Delta x^{H_{h}}$ where $\epsilon(\Delta x)$ is a UM field, $a_{h}=1 / 3$ and $H_{h}=1 / 3$. An anisotropy between horizontal and vertical shifts is accounted for by considering a different scaling relation in the vertical $\Delta u(\Delta z) \approx \epsilon(\Delta z)^{a_{v}} \Delta z^{H_{v}}$ with $\epsilon(\Delta z)$ again a UM field, $a_{v}=1 / 5$ and $H_{v}=3 / 5$.

Relying on this existing framework, the following formulas are used to generate a 3D wind field at a distance $\Delta x, \Delta y$ $\left(\Delta l=\sqrt{\Delta x^{2}+\Delta y^{2}}\right), \Delta z$ from a point $(x, y, z)$ :

$$
\begin{aligned}
& u_{x}(x+\Delta x, y+\Delta y, z+\Delta z, t)=u_{x}(x, y, z, t)+c_{\epsilon_{x}} \epsilon_{x}(\Delta x, \Delta y, t)^{a_{h}} \Delta l^{H_{h}}+c_{\theta_{x}} \theta_{x}(\Delta z, t)^{a_{v}} \Delta z^{H_{v}} \\
& u_{y}(x+\Delta x, y+\Delta y, z+\Delta z, t)=u_{y}(x, y, z, t)+c_{\epsilon_{y}} \epsilon_{y}(\Delta x, \Delta y, t)^{a_{h}} \Delta l^{H_{h}}+c_{\theta_{y}} \theta_{y}(\Delta z, t)^{a_{v}} \Delta z^{H_{v}} \\
& u_{z}(x+\Delta x, y+\Delta y, z+\Delta z, t)=u_{z}(x, y, z, t)+c_{\epsilon_{z}} \epsilon_{z}(\Delta x, \Delta y, t)^{a_{h}} \Delta l^{H_{h}}+c_{\theta_{z}} \theta_{z}(\Delta z, t)^{a_{v}} \Delta z^{H_{v}}
\end{aligned}
$$

The fields $\epsilon$ (i.e. the ones for the horizontal shift) are simulated in space time with a size $729 \times 729$ x 64 using discrete UM cascades and Eq. 10 to obtain either positive or negative values. A simple anisotropy between space and time is accounted through a scaling anisotropy coefficient $H_{t}$. In such framework, when the spatial scale of the data is changed by a ratio of $\lambda_{x y}$, then the temporal scale should be changed by a factor of $\lambda_{t}=\lambda_{x y}^{H_{t}}$. $H_{t}$ is expected to be equal to 1/3 (Marsan et al., 1996), hence when the spatial scale is multiplied by 3 , the temporal scale should be multiplied by 2 (i.e. $3^{1-1 / 3} \approx 2.08$ ) (Biaou et al., 2005; Gires et al., 2014). These fields are assumed to cover an area of size $40 \mathrm{~km} \times 40 \mathrm{~km} \times 1024 \mathrm{~s}$, which is needed for drift of $0.1 \mathrm{~mm}$ drops during their fall when wind is strong. It means that a voxel is of size is $53 \mathrm{~m} \times 53 \mathrm{~m} \times 16 \mathrm{~s}$.

The fields $\theta$ (i.e. the ones for the vertical shift) are of size $512 \times 64$ covering a physical are of $1600 \mathrm{~m} \times 1024 \mathrm{~s}$, meaning that a pixel size $3 m \times 16 \mathrm{~s}$. All $\mathrm{UM}$ fields are simulated with $\alpha=1.7, C_{1}=0.2$ as in the previous section.

The strong assumption that there are no correlations between the differences along the horizontal axis and the vertical one is done ! No correlation between the shifts for the various components are also assumed. Finally, at any point x,y,z,t a bi or trilinear interpolation is implemented to obtain the value of the field from the nearest points. The value of the prefactor were set to $c_{\epsilon_{x}}=c_{\epsilon_{y}}=0.3, c_{\epsilon_{z}}=0.1$ and $c_{\theta_{x}}=c_{\theta_{y}}=c_{\theta_{z}}=0.01$, through an heuristic approach of trial and error to get some realistic fluctuations. In the future, it would obviously be needed to tune them to local wind properties. However such tuning is outside the scope of this section, which aims more at being a proof of concept. 


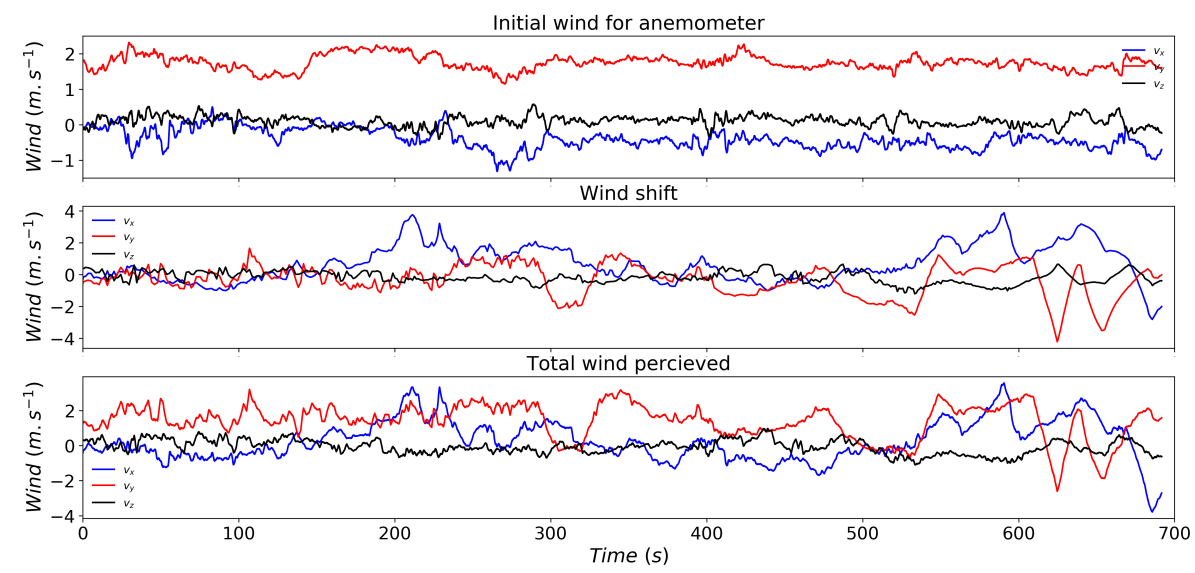

Figure 6. Temporal evolution with $0.01 s$ time steps of the wind data from 3D sonic anemometer (a), the wind shift (b) and the total wind perceived (c) by the $0.5 \mathrm{~mm}$ drop falling from the position $(0,0,1500)$ during the low wind event. (c) is actually the wind input used to obtain the trajectory of Fig. 7.

\subsection{Illustration}

In order to illustrate the suggested process, let us consider a $0.5 \mathrm{~mm}$ drop during the low wind event. Its initial position is $(0,0,1500)$ in $m$. It is 'dropped' with no horizontal velocity and a vertical one equal to its terminal fall one. The anemometer is assumed to be located at at $(0,0,100) \mathrm{m}$. Then Eq. 5 is implemented. At each time step the local wind is assessed using the methodology described in the previous paragraphs.

The actual total wind perceived by the drop (i.e. input in Eq. 5) is recorded and displayed in last row of Fig. 7. It corresponds to the sum of the wind from the anemometer (first row in Fig. 7) plus a wind shift field (middle row in Fig. 7). This yields to a given trajectory in space which is shown in Fig. 7. Projected trajectory on the plans (x,y) and (x,z) are also shown. This trajectory exhibits a non linear complex pattern which results from the turbulent nature of the wind.

\subsection{Sensitivity to the wind shift field}

The process to generate an estimation of a 3D wind field is actually stochastic through the UM fields $\epsilon$ and $\theta$ used in Eq. 11 . In this section, the sensitivity to the given realization of the process is discussed. In order to achieve that, 10 wind samples are generated and the corresponding trajectories for drops of size $0.5,1,2$ and $3 \mathrm{~mm}$ are computed.

For the low wind event, the projected trajectories are displayed in Fig. 8.a and b. The position of the drop when they reach the ground is in Fig. 8.c. The spread of the drops strongly depends on their size, with a decrease as drop size increases. Indeed $\Delta x\left(x_{\max }-x_{\min }\right)$ is equal to $1238,591,415$ and $404 \mathrm{~m}$ for drop of size $0.5,1,2$ and $3 \mathrm{~mm}$ respectively. For $\Delta y$ the values are 2123, 897, 461 and $322 \mathrm{~m}$. Such decrease is due to a combination of the fact that smaller drops are more subject to wind fluctuations (Section 3), and that they spend more time in the atmosphere (section 2) before they reach the ground. Similar 
https://doi.org/10.5194/amt-2021-434

Preprint. Discussion started: 7 February 2022

(C) Author(s) 2022. CC BY 4.0 License.

(c) (i)

\section{Atmospheric \\ Measurement \\ Techniques}

Discussions

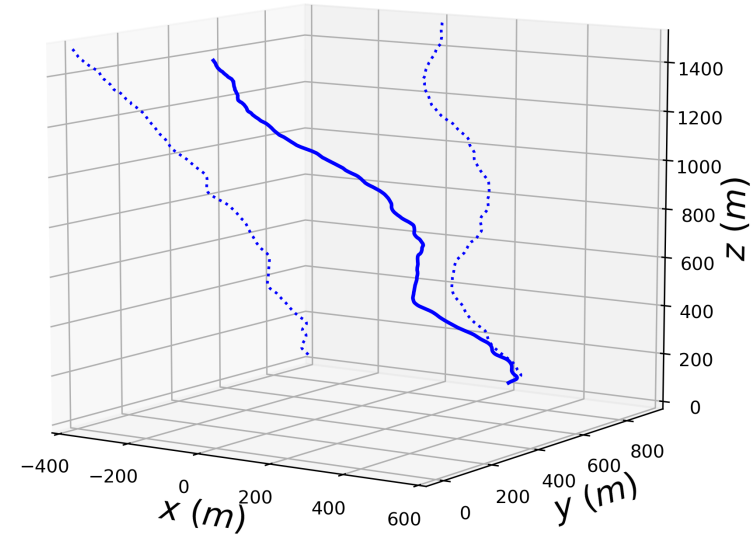

Figure 7. Trajectory (solid line) of a $0.5 \mathrm{~mm}$ drop in a turbulent wind field for the low wind event. The dotted lines correspond to the trajectory projected on the $(\mathrm{x}, \mathrm{z})$ and $(\mathrm{y}, \mathrm{z})$ plan
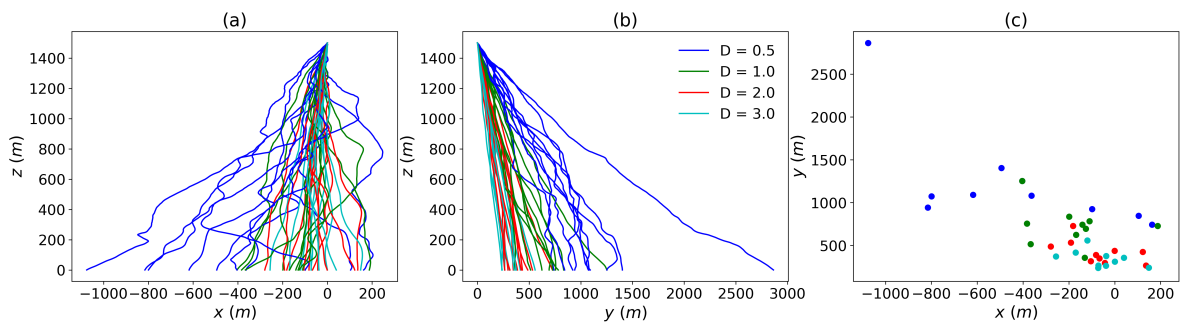

Figure 8. (a) and (b) Trajectories of drops of various sizes falling from the $(0,0,1500)$ position, projected on the (x,z) and (y,z) plan respectively. Low wind event is used. The 10 curves correspond to different realizations of the wind shift field. (c) Position of the ground impact of the various drops

trends are retrieved for the strong wind event (Fig. 9) with a stronger absolute shift. In that case the value for $\Delta x$ are 890,868 , 500 and $448 m$ respectively. For $\Delta y$ they are 2382, 1003, 568 and $448 \mathrm{~m}$.

\subsection{Illustration of impact on rainfall retrieval with weather radars}

In this last section, initial investigations toward understanding the consequence of previous work on quantitative rainfall measurement with weather radars are carried out. Indeed, weather radar measure rainfall at a given altitude while hydrometeorologist are interested about rainfall at ground level. During their fall, significant shift can occur. In order to study it, the following process is implemented. During five minutes, one rainfall drop is dropped every $15 s$ from a random position withing a voxel of size $100 \mathrm{~m}$ centered on $(0,0,1450) \mathrm{m}$. Hence it covers a total duration of $5 \mathrm{~min}$. The trajectories and positions on 

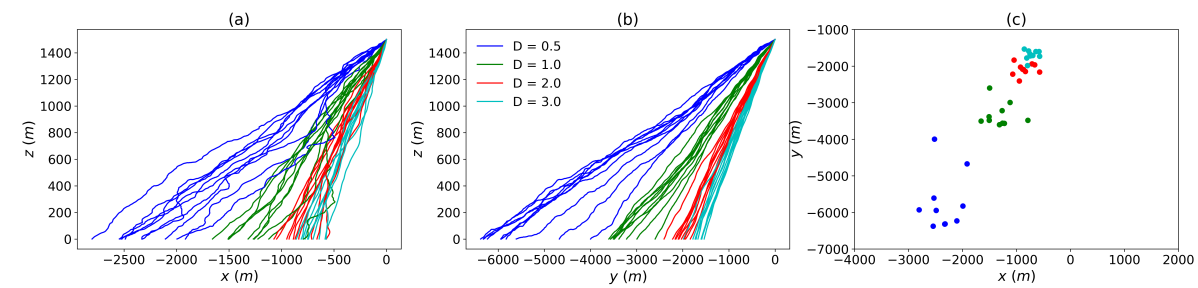

Figure 9. Same as in Fig. 8 but for the strong wind event
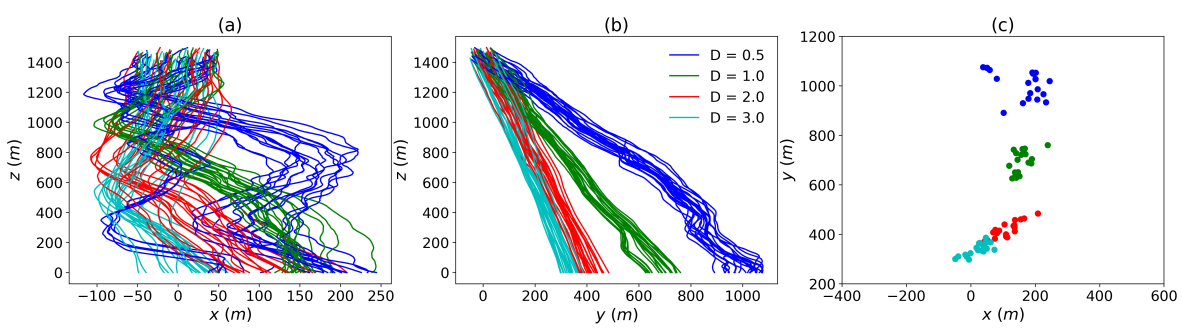

Figure 10. (a) and (b) Trajectories of drops of various sizes falling from a 100 m cubic voxel centered on $(0,0,1450)$ position, projected on the $(\mathrm{x}, \mathrm{z})$ and $(\mathrm{y}, \mathrm{z})$ plan respectively. Low wind event is used. For each size, the 20 drops are dropped every $15 \mathrm{~s}$ (hence over a total duration of $5 \mathrm{~min}$ ). A single realization of the wind shift field is used. (c) Position of the ground impact of the various drops

the ground of the drops is then studied. This enables to basically mimic the measurement of a weather radar at its typical gate size and temporal resolution.

Fig. 10 displays the trajectories and ground impact location in the case of the low wind event for a given realisation of the stochastic wind shift. A shift of more than $1 \mathrm{~km}$ for small drops is found and more than $300 \mathrm{~m}$ for $3 \mathrm{~mm}$ drops. As noted in the previous section, the spread of drops at ground level tends to decrease with increasing drop size. Indeed $\Delta x\left(x_{\max }-x_{\min }\right)$ is equal to 206, 119, 165 and $121 \mathrm{~m}$ for drop of size 0.5, 1, 2 and $3 \mathrm{~mm}$ respectively. For $\Delta y$ the values are 184, 134, 112 and $88 \mathrm{~m}$. For the strong wind event (Fig. 11), shifts of more than $6 \mathrm{~km}$ and $1.5 \mathrm{~km}$ are reported for drops of size $0.5 \mathrm{~mm}$ and 3 $\mathrm{mm}$ are retrieved. Similar results as for the low wind event are found with regards to the spread. The corresponding figures are of 665, 450, 284 and $307 m$ for $\Delta x$, and of 819, 664, 429 and 420 for $\Delta y$.

As previously pointed out, this spread is due to the fact that smaller drop spend more time in the atmosphere and are more sensitive to wind fluctuations. Indeed the duration of fall from $1500 \mathrm{~m}$ to the ground at $0 \mathrm{~m}$ is equal to 716, 378, 238 and 192 $s$ for drops of size $0.5,1,2$ and $3 \mathrm{~mm}$ respectively. Given that high resolution radar pixels are typically of size few hundred meters, one should note that drops within a given voxel at measurement height can reach ground within an area of size $3 \mathrm{~km} x$ $6 \mathrm{~km}$. Even within a drop diameters class, shifts are of few radar pixels. Given that drop size distribution also varies, such shift can significantly affect rainfall retrieval, even in low wind conditions. 

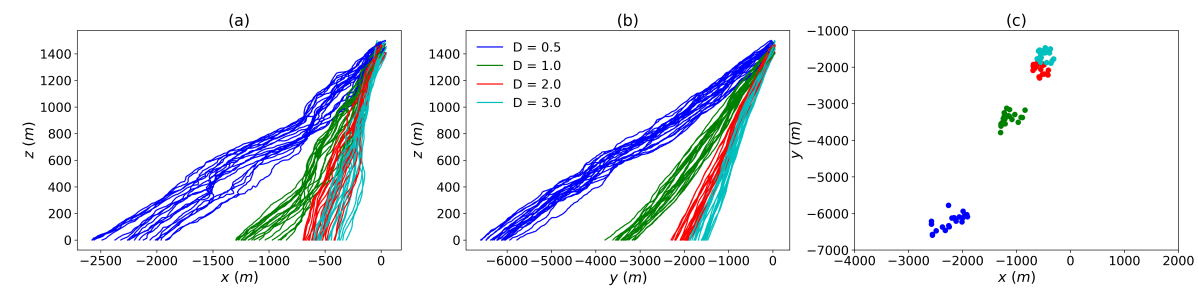

Figure 11. Same as in Fig. 10 but for the strong wind event

\section{Conclusions}

In this paper we aimed at better understanding the behaviour of rainfall drops falling from typically $1500 \mathrm{~m}$. In a first step we developed a new approach to compute the drag coefficient accounting for drop oblateness and findings in fluid mechanics. This was validated for drop of equivolumic size of up to $4 \mathrm{~mm}$ through the comparison between retrieved terminal fall velocity and commonly used formula.

Then the temporal evolution of horizontal drop velocity under turbulent wind constraints was studied. It appears that multifractal features of the input wind are simply transferred to drop velocity with an additional fractional integration and slight time shift. UM parameter $\alpha$ and $C_{1}$ are basically conserved while $H$ is increased. The increase ranges from 0.1 for $0.1 \mathrm{~mm}$ size drop to 0.8 for drops of size 1-1.5 $\mathrm{mm}$. It remains rather constant for larger drops.

Finally the trajectories of drop of various size falling form $1500 \mathrm{~m}$ was studied as a proof of concept. For this, $100 \mathrm{~Hz}$ anemometer data was used and an approach to simulate realistic fluctuations of wind in space was developed. It notably enables to analyse how drop are shifting during their fall between their location measurement by weather radars and ground impact. For a strong wind event, drops located within a radar gate in altitude during $5 \mathrm{~min}$ are spread on the ground over an area of size few kilometers. Spread for drops of a given diameter are found to cover few radar pixels.

In order to explore further the consequences of these findings on quantitative rainfall estimation with weather radars, further investigations are needed. More precisely (i) the model to simulate wind fluctuations should be improved, notably to tune the prefactors to local conditions; (ii) space-time outputs of numerical weather prediction models could also be tested; (iii) actual drop size distribution should be used to assess better the impact for ground estimation of precipitation; (iv) longer period of time should be tested. For the two last points, data is available within the RW-Turb project.

Acknowledgements. The authors gratefully acknowledge partial financial support from the Chair "Hydrology for Resilient Cities" (endowed by Veolia) of Ecole des Ponts ParisTech, the Île-de-France region RadX@IdF Project, and the ANR JCJC RW-Turb project (ANR-19-CE050022-01) 
Atlas, D., Srivastava, R. C., and Sekhon, R. S.: Doppler radar characteristics of precipitation at vertical incidence, Reviews of Geophysics, 11, 1-35, https://doi.org/https://doi.org/10.1029/RG011i001p00001, 1973.

Baheri, G.: Numerical and experimental investigation of particle terminal velocity and aggregation in volcanic plumes. Thèse de doctorat no. Sc. 4844, Ph.D. thesis, Univ. Genève, 2015.

Beard, K. V.: Terminal velocity adjustment for cloud and precipitation aloft, J. Atmos. Sci, 34, 1293-1298, 1977.

Best, A. C.: Empirical formulae for the terminal velocity of water drops falling through the atmosphere, Quarterly Journal of the Royal Meteorological Society, 76, 302-311, https://doi.org/https://doi.org/10.1002/qj.49707632905, 1950.

Biaou, A., Chauvin, F., Royer, J.-F., and Schertzer, D.: Analyse multifractale des précipitations dans un scénario GIEC du CNRM, Note de centre GMGEC, CNRM, 101, 45, 2005.

Blocken, B., Stathopoulos, T., Carmeliet, J., and Hensen, J. L.: Application of computational fluid dynamics in building performance simulation for the outdoor environment: an overview, Journal of Building Performance Simulation, 4, 157-184, https://doi.org/10.1080/19401493.2010.513740, 2011.

Bringi, V., Thurai, M., and Baumgardner, D.: Raindrop fall velocities from an optical array probe and 2-D video disdrometer, Atmospheric Measurement Techniques, 11, 1377-1384, https://doi.org/10.5194/amt-11-1377-2018, 2018.

Collier, C.: The impact of wind drift on the utility of very high spatial resolution radar data over urban areas, Physics and Chemistry of the Earth, Part B: Hydrology, Oceans and Atmosphere, 24, 889-893, https://doi.org/https://doi.org/10.1016/S1464-1909(99)00099-4, 1999.

Dai, Q., Han, D., Rico-Ramirez, M. A., and Islam, T.: The impact of raindrop drift in a three-dimensional wind field on a radar-gauge rainfall comparison, International Journal of Remote Sensing, 34, 7739-7760, https://doi.org/10.1080/01431161.2013.826838, 2013.

Dai, Q., Yang, Q., Han, D., Rico-Ramirez, M. A., and Zhang, S.: Adjustment of Radar-Gauge Rainfall Discrepancy Due to Raindrop Drift and Evaporation Using the Weather Research and Forecasting Model and Dual-Polarization Radar, Water Resources Research, 55, 9211-9233, https://doi.org/https://doi.org/10.1029/2019WR025517, 2019.

Fitton, G., Tchiguirinskaia, I., Schertzer, D., and Lovejoy, S.: Scaling Of Turbulence In The Atmospheric Surface-Layer: Which Anisotropy?, Journal of Physics: Conference Series, 318, 072 008, https://doi.org/10.1088/1742-6596/318/7/072008, 2011.

Gires, A., Tchiguirinskaia, I., Schertzer, D., Schellart, A., Berne, A., and Lovejoy, S.: Influence of small scale rainfall variability on standard comparison tools between radar and rain gauge data, Atmospheric Research, 138, 125-138, http://www.sciencedirect.com/science/article/ pii/S0169809513003293, 2014.

Gires, A., Tchiguirinskaia, I., and Schertzer, D.: Blunt extension of discrete universal multifractal cascades: development and application to downscaling, Hydrological Sciences Journal, 0, 1-17, https://doi.org/10.1080/02626667.2020.1736297, 2020.

Gires, A., Jose, J., Tchiguirinskaia, I., and Schertzer, D.: Data for : "Three months of combined high resolution rainfall and wind data collected on a wind farm", https://doi.org/10.5281/zenodo.5801900, 2021.

Hölzer, A. and Sommerfeld, M.: New simple correlation formula for the drag coefficient of non-spherical particles, Powder Technology, 184, 361-365, https://doi.org/https://doi.org/10.1016/j.powtec.2007.08.021, 2008.

Kruger, A. and Krajewski, W. F.: Two-Dimensional Video Disdrometer: A Description, Journal of Atmospheric and Oceanic Technology, 19, 602-617, http://dx.doi.org/10.1175/1520-0426(2002)019<0602:TDVDAD>2.0.CO;2, 2002.

345 Lack, S. A. and Fox, N. I.: An examination of the effect of wind-drift on radar-derived surface rainfall estimations, Atmospheric Research, 85, 217-229, https://doi.org/https://doi.org/10.1016/j.atmosres.2006.09.010, 2007. 
Lauri, T., Koistinen, J., and Moisseev, D.: Advection-Based Adjustment of Radar Measurements, Monthly Weather Review, 140, 1014 1022, https://doi.org/10.1175/MWR-D-11-00045.1, 2012.

Lavallée, D., Lovejoy, S., and Ladoy, P.: Nonlinear variability and landscape topography: analysis and simulation, in: Fractas in geography, edited by de Cola, L. and Lam, N., pp. 171-205, Prentice-Hall, 1993.

Lazarev, A., Schertzer, D., Lovejoy, S., and Chigirinskaya, Y.: Unified multifractal atmospheric dynamics tested in the tropics: part II, vertical scaling and Generalized Scale Invariance, Nonlinear Processes in Geophysics, 1, 115-123, 1994.

Lhermitte, R. M.: Cloud and precipitation remote sensing at $94 \mathrm{GHz}$, Geoscience and Remote Sensing, IEEE Transactions on, 26, 207-216, 1988.

355 Marsan, D., Schertzer, D., and Lovejoy, S.: Causal space-time multifractal processes: Predictability and forecasting of rain fields, J. Geophys. Res., 101, 26333-26346, 1996.

Mittermaier, M. P., Hogan, R. J., and Illingworth, A. J.: Using mesoscale model winds for correcting wind-drift errors in radar estimates of surface rainfall, Quarterly Journal of the Royal Meteorological Society, 130, 2105-2123, https://doi.org/https://doi.org/10.1256/qj.03.156, 2004.

Montero-Martinez, G. and Garcia-Garcia, F.: On the behaviour of raindrop fall speed due to wind, Quarterly Journal of the Royal Meteorological Society, 2016.

Pedersen, H. S. and Hasholt, B.: Influence of wind speed on rainsplash erosion, CATENA, 24, $39-54$, https://doi.org/https://doi.org/10.1016/0341-8162(94)00024-9, 1995.

Sandford, C.: Correcting for wind drift in high resolution radar rainfall products: a feasibility study, Journal of Hydrology, 531, 284-295, https://doi.org/https://doi.org/10.1016/j.jhydrol.2015.03.023, hydrologic Applications of Weather Radar, 2015.

Schertzer, D. and Lovejoy, S.: Physical modelling and analysis of rain and clouds by anisotropic scaling and multiplicative processes, J. Geophys. Res., 92, 9693-9714, 1987.

Schertzer, D. and Lovejoy, S.: From scalar cascades to lie cascades: joint multifractal analysis of rain and cloud processes, in: Space/time variability and interdependence for various hydrological processes, edited by Feddes, R., pp. 153-173, Cambridge University Press, 1995.

Schertzer, D. and Lovejoy, S.: Universal multifractals do exist!: Comments, Journal of Applied Meteorology, 36, 1296-1303, <GotoISI>: //WOS:A1997XU62400016, j. Appl. Meteorol., 1997.

Schertzer, D. and Tchiguirinskaia, I.: A Century of Turbulent Cascades and the Emergence of Multifractal Operators, Earth and Space Science, 7, e2019EA000 608, https://doi.org/10.1029/2019EA000608, e2019EA000608 10.1029/2019EA000608, 2020.

Stout, J. E., Arya, S. P., and Genikhovich, E. L.: The Effect of Nonlinear Drag on the Motion and Settling Velocity of Heavy Particles, Journal of the Atmospheric Sciences, 52, 3836-3848, https://doi.org/10.1175/1520-0469(1995)052<3836:TEONDO>2.0.CO;2, 1995.

Thurai, M., Huang, G. J., Bringi, V. N., Randeu, W. L., and Sch ̃̃đnhuber, M.: Drop Shapes, Model Comparisons, and Calculations of Polarimetric Radar Parameters in Rain, Journal of Atmospheric and Oceanic Technology, 24, 1019-1032, http://dx.doi.org/10.1175/ JTECH2051.1, 2007.

Thurai, M., Schonhuber, M., Lammer, G., and Bringi, V.: Raindrop shapes and fall velocities in "turbulent times", Advances in Science and

Research, 16, 95-101, https://doi.org/10.5194/asr-16-95-2019, 2019.

Tian, L., Zeng, Y.-J., and Fu, X.: Velocity Ratio of Wind-Driven Rain and Its Application on a Transmission Tower Subjected to Wind and Rain Loads, Journal of Performance of Constructed Facilities, 32, 04018 065, https://doi.org/10.1061/(ASCE)CF.1943-5509.0001210, 2018.

White, F.: Viscous Fluid Flow, McGraw-Hill, 1974. 